\title{
Angiotensin II Type-I Receptor Blocker, Candesartan, Improves Brachial-Ankle Pulse Wave Velocity Independent of Its Blood Pressure Lowering Effects in Type 2 Diabetes Patients
}

\author{
Hideto Ishii ${ }^{1,2}$, Toyohiro Tsukada ${ }^{2}$ and Masayuki Yoshida ${ }^{2}$
}

\begin{abstract}
Background Hypertension in diabetic patients has been shown to accelerate the progression of atherosclerosis and angiotensin II type-I (AT1) receptor blocker (ARB) has been shown to have anti-atherosclerosis action independent of its action on blood pressure.

Methods and Results Type 2 diabetic patients with hypertension received either ARB (candesartan), or a calcium channel blocker (CCB; amlodipine or nifedipine) for 12 weeks. Though clinical parameters such as blood glucose, HbA1c, and systolic and diastolic pressure were not significantly changed between the two groups, brachial-ankle pulse wave velocity (baPWV) was significantly reduced in the candesartan group when compared to CCB groups. Moreover, candesartan treatment exhibited a tendency of reduction in inflammation markers such as high sensitive C-reactive protein (hsCRP) and interleukin-6 (IL-6).

Conclusion We conclude that candesartan may improve vascular inflammation independent of its effect on hypertension in diabetes, thus suggesting its efficacy in diabetic patients.
\end{abstract}

Key words: AT1 receptor blocker, pulse wave velocity, type 2 diabetes mellitus

(Inter Med 47: 2013-2018, 2008)

(DOI: 10.2169/internalmedicine.47.1162)

\section{Introduction}

Diabetes mellitus is manifested by injuries in various vascular beds, which determine its prognosis. Although atherosclerosis is not specific for diabetes mellitus, it is the most fatal diabetic complication. High blood pressure with diabetes mellitus accelerates the progression of atherosclerosis (1). The renin-angiotensin system (RAS) has been shown to be activated in diabetes mellitus (2). RAS also plays a role in the pathogenesis of atherosclerosis (3-5). Prior studies provide evidence that an angiotensin II type-I (AT1) receptor blocker (ARB) shuts off RAS via blockade of binding angiotensin II to AT1 receptor followed by a reduction in cardiovascular events.

Inflammation markers, such as high sensitivity C-reactive protein (hsCRP) (6) and interleukin-6 (IL-6) (7) are often increased in patients with cardiovascular disease. Leukocyteendothelial cell adhesion is regarded as one of the manifestations of inflammation and atherosclerosis (8). Adhesion molecules, such as E-selectin, intracellular adhesion molecule-1 (ICAM-1), vascular cell adhesion molecule-1 (VCAM-1) are upregulated in endothelial cells in atherosclerosis (9).

Though the effect of ARB in anti-inflammation has been postulated previously the mechanisms are not fully understood. For example, Wu L et al showed stimulation of the AT2 receptor after AT1 blockade by ARB inhibited inflammatory vascular injury (10). Cianchetti et al described that ARB modulates TNF-alpha-induced VCAM-1 expression in vascular endothelium (11).

On the other hand, a calcium channel blocker (CCB) is frequently used for the treatment of hypertension. The effectiveness of candesartan has been demonstrated in the re-

${ }^{1}$ Life Science and Bioethics Research Center, Tokyo Medical and Dental University, Tokyo and ${ }^{2}$ Department of Internal Medicine, Tamananbu Regional Hospital, Tama

Received for publication March 26, 2008; Accepted for publication August 14, 2008

Correspondence to Masayuki Yoshida, masavasc@tmd.ac.jp 
cently reported CASE-J study in Japan (12). This study showed that though CCB inhibited death more strongly compared to ARB in the early period, ARB surpassed CCB in death reduction after about 18 months from the starting point. Considering that the reduction of blood pressure was almost identical between $\mathrm{ARB}$ and $\mathrm{CCB}, \mathrm{ARB}$ seemed to have an anti-atherosclerotic effect independent of its blood pressure reduction.

Brachial-ankle pulse wave velocity (baPWV) is a recently developed method of assessing atherosclerosis by measuring the stiffness of vascular wall. Previous reports showed positive associations between baPWV and atherosclerotic disease (13-15).

In this study, we attempted to compare the antiatherosclerotic effect with $\mathrm{ARB}$ and $\mathrm{CCB}$ in diabetes mellitus using non-invasive baPWV and inflammation markers in serum, such hsCRP and IL-6.

\section{Study Design and Procedures}

\section{Subjects}

This study was performed on 22 type 2 diabetes patients according to the diagnostic criteria of the Japan Diabetes Society with high blood pressure (systolic blood pressure $\geqq$ $140 \mathrm{mmHg}$ and/or diastolic blood pressure $\geqq 90 \mathrm{mmHg}$ ). Patients without prior medication for hypertension were recruited. However, the usage of any anti-diabetic medicine including insulin therapy was permitted. Their diabetic microangiopathies were as follows: retinopathy was nothing or simple, nephropathy did not exceed early stage urinary albu$\min <300 \mathrm{mg} / \mathrm{g}$. Cr, and neuropathy did not show an obvious symptom. No obvious diabetic angiopathies were observed in this study.

They were randomly divided into three groups, and assigned to receive candesartan, amlodipine or nifedipine. The patients were followed up for 90 days without changing the drugs. The objective of the study was explained to all patients enrolled in the study, and their informed consent was obtained prior to the start of the study.

\section{Medication and data collection}

Enrolled patients were randomized to either $8 \mathrm{mg}$ of candesartan (Blopress) $(\mathrm{n}=11)$ as $\mathrm{ARB}, 5 \mathrm{mg}$ of amplodipine (Norvasc) $(\mathrm{n}=6)$ as $\mathrm{CCB}$ or $40 \mathrm{mg}$ (Adalat CR) of nifedipine $(n=5)$ as CCB. Additional antihypertensive medications were not used during the examination period. baPWV, hsCRP, IL-6, VCAM-1, and HbA1c of patients were measured at the starting point and at 90 days after randomization.

\section{baPWV measurement}

PWV was measured using a Form PWV/ABI (ColinOmron, Tokyo, Japan). Detailed measurement method was described previously (16). Briefly, the patient was allowed to rest for 5 minutes and examined in the supine position.
After attaching cuffs and electrodes, blood pressure, baPWV, and ABI (ankle-brachial index) were measured.

\section{Blood sampling}

hsCRP (by latex-enhanced immunonephelometrics), IL-6 (by CLEIA), VCAM-1 (by ELISA), and HbA1c(by latex agglutination) were measured by SRL Co., Ltd (Tokyo).

\section{Adhesion Assay under static condition}

Static adhesion assays were carried out as previously described in detail (17). In brief, THP-1 cells were prelabeled with BCECF-AM for 20 minutes at $37^{\circ} \mathrm{C}$, and then placed on TNF- $\alpha$ stimulated HUVECs plated in a 96-well microtiter culture plate and incubated for 10 minutes at room temperature. The fluorescent intensity of the monolayerassociated THP-1 cells was quantitated using a fluorescent plate reader (Applied Biosystems, Foster City, CA, USA).

\section{Statistical analysis}

All the numerical data were expressed as means \pm standard deviations (S. D.). Matched-paired $t$-test was used for comparisons between the groups. Differences at $\mathrm{p}<0.05$ were considered to be statistically significant.

\section{Results}

\section{Patient characteristics}

As shown in Table 1, there were no significant differences in age, body mass index (BMI), and diabetes control (fasting plasma glucose and $\mathrm{HbA} 1 \mathrm{c}$ ) between $\mathrm{ARB}$ and $\mathrm{CCB}$ groups. Systolic and diastolic blood pressure was not significantly changed throughout the experimental period between the two groups (Fig. 1a, b).

\section{Candesartan inhibits baPWV independent blood pressure lowering effect}

Reductions of baPWV (rPWV) from baseline after treatment were calculated. rPWV was enhanced by ARB, candesartan, compared to CCB (ARB -636.64+/-383.91 cm/s, CCB $-172.99+/-150.87 \mathrm{~cm} / \mathrm{s} \mathrm{p}<0.05)($ Fig. 2). In this study, two CCB compounds amlodipine (AD) and nifedipine (ND) were used. Candesartan (CS) boosted rPWV significantly compared to $\mathrm{AD}(\mathrm{CS}-636.64+/-383.91 \mathrm{~cm} / \mathrm{s}$, $\mathrm{AD}$ $-156.55+/-137.54 \mathrm{~cm} / \mathrm{s} \mathrm{p}<0.01)$ or $\mathrm{ND}(\mathrm{CS}-636.64+/-383.91$ $\mathrm{cm} / \mathrm{s}, \mathrm{ND}-189.42+/-174.68 \mathrm{~cm} / \mathrm{s} \mathrm{p}<0.05$ ) (data not shown).

\section{Anti-inflammatory effect of candesartan in vivo and in vitro}

We measured high sensitivity C-reactive protein (hsCRP), interleukin-6 (IL-6), and VCAM-1 in patient's blood samples. As shown in Fig. 3a, candesartan, reduced hsCRP compared to CCB (ARB -1784.00+/-1632.02 mg/mL, CCB $-535.00+/-1005.85 \mathrm{mg} / \mathrm{mL} \mathrm{p}=0.095)$. IL-6 also yielded a similar result (ARB -1.2+/-1.12 pg/mL, CCB $0.93+/-2.20$ $\mathrm{pg} / \mathrm{mL} \mathrm{p}=0.09$ ) (data not shown). On the other hand, the re- 
Table 1. Comparison of General Characteristics and Diabetic Marker betweem ARB and CCB Group

\begin{tabular}{|c|c|c|c|}
\hline & ARB & CCB & p-value \\
\hline age (years old) & $68.4 \pm 9.5$ & $68.0 \pm 6.0$ & 0.467 \\
\hline BP (mmHg) & $163 / 89 \pm 14 / 6$ & $167 / 91 \pm 23 / 6$ & $\begin{array}{l}\text { SBP:0.700 } \\
\text { DBP:0.640 }\end{array}$ \\
\hline baPWV(cm/s) & $2007.8 \pm 473.5$ & $1985.7 \pm 638.4$ & 0.924 \\
\hline BMI & $23.9 \pm 4.0$ & $23.5 \pm 2.9$ & 0.851 \\
\hline FPG & $190.5 \pm 37.7$ & $184.5 \pm 40.1$ & 0.795 \\
\hline HbA1c (\%) & $8.1 \pm 0.8$ & $7.1 \pm 0.8$ & 0.110 \\
\hline
\end{tabular}

All data show patient's profile at start point.

BP:blood pressure.

SBP:systolic blood pressure.

DBP:diastolic blood pressure.

BMI: body mass index

FPG: fasting plasma glucose

$\mathrm{p}$-value was calculated by t-test

(a)

$\mathrm{mmHg}$

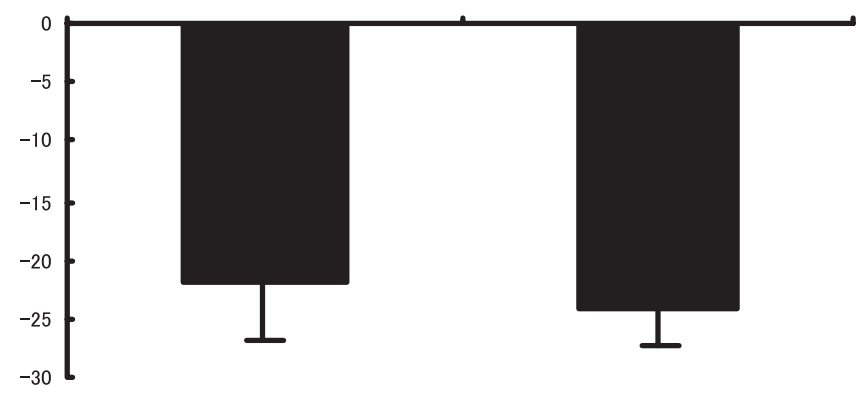

ARB

(b)

$\mathrm{mmHg}$

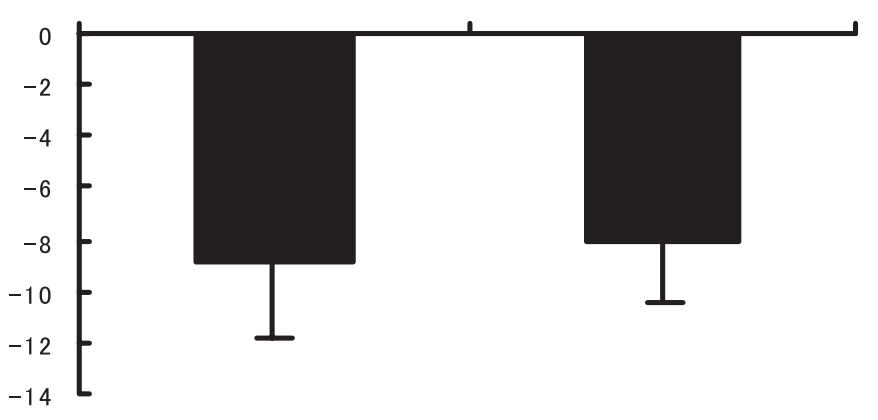

ARB

CCB

Figure 1. Reduction of blood pressure is almost equal between ARB group and CCB group (a) reduction of systolic blood pressure (b) reduction of diastolic blood pressure. $\mathrm{cm} / \mathrm{s}$

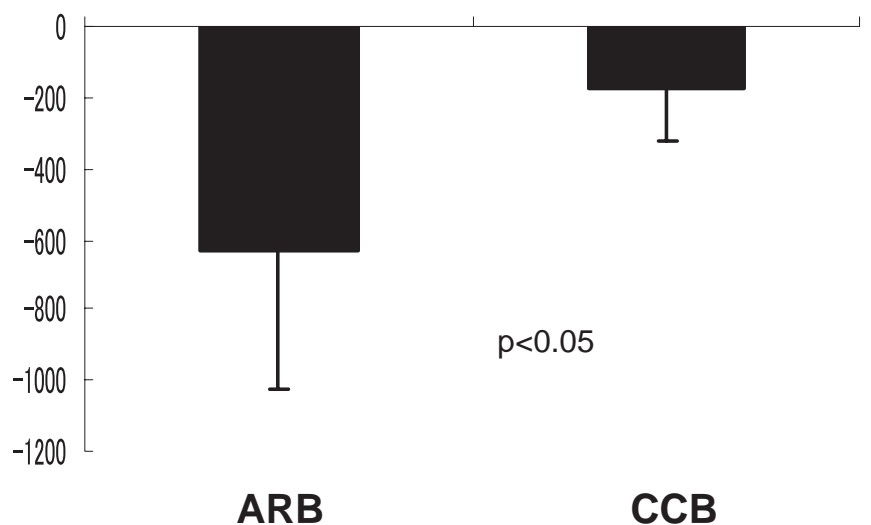

Figure 2. Reduction levels of baPWV (rPWV). ARB boosted reduction of baPWV.

duction of VCAM-1 was not significantly changed (ARB $-126.00+/-215.52 \mathrm{ng} / \mathrm{mL}, \mathrm{CCB}-7.50+/-196.92 \mathrm{ng} / \mathrm{mL} \mathrm{p}=$ 0.22) (Fig. 3b).

Next, adhesion assay was performed to examine the antiadhesive function of candesartan in vitro. Human umbilical vein endothelial cells (HUVECs) were stimulated with $5 \mathrm{ng} /$ $\mathrm{ml}$ tumor necrosis factor-alpha (TNF- $\alpha$ ) in the presence or absence of pretreatment. As shown in Fig. 3c, ARB inhibited monocytic cell adhesion to activated HUVECs in static adhesion assay (TNF- $\alpha$ 271.25+/-40.58 R. F. U., ARB 115.25+/-9.07 R. F. U. p<0.01).

\section{Candesartan improves HbA1c level}

Though the medication for diabetes mellitus was not changed during this study period, ARB, candesartan, tended 
(a)

$\mathrm{ng} / \mathrm{mL}$

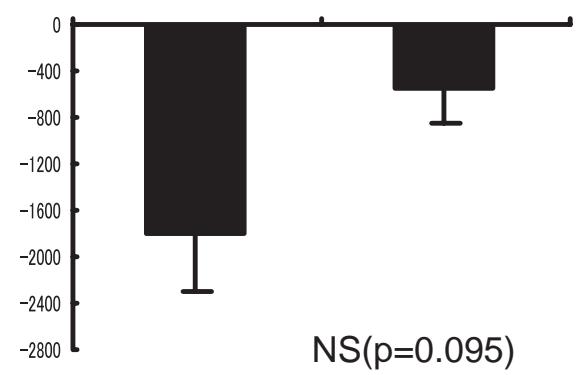

ARB
hsCRP

$N S(p=0.095)$ (b)

$\mathrm{ng} / \mathrm{mL}$
VCAM-1

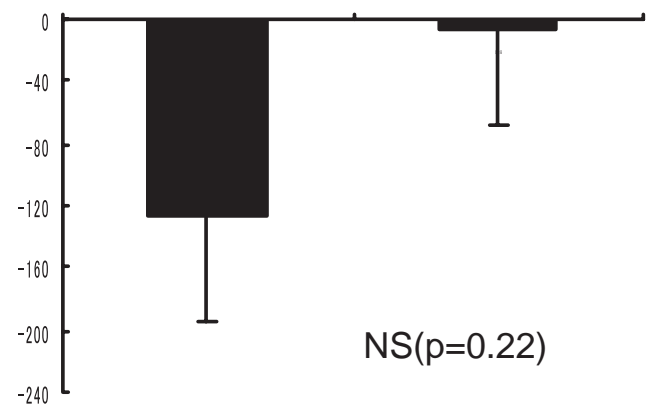

ARB

CCB

(c)

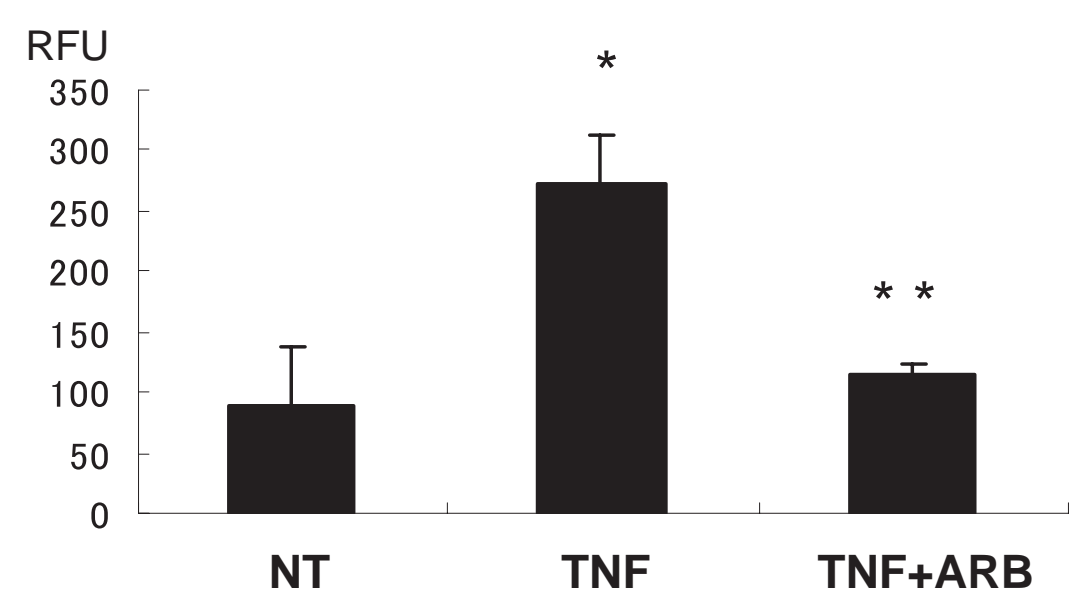

Figure 3. Inflammatory marker change by ARB or CCB ARB inhibited the expression of inflammatory markers. (a) hsCRP (b) VCAM-1 (c) static adhesion assay: NT means no treatment, TNF means TNF- $\alpha$ stimulation, and TNF+CS means TNF- $\alpha$ stimulation with $5 \mu$ M of candesartan. $* \mathbf{p}<0.01$ vs. NT, **p $<0.01$ vs. TNF.

to reduce $\mathrm{HbA} 1 \mathrm{c}$ level compared to $\mathrm{CCB}$. (ARB $-1.10+/-1.15 \%$, CCB $0.3+/-1.67 \%$ p=0.12) (Fig. 4). However, candesartan significantly decreased HbA1c compared to nifedipine (reduction: ARB $-1.10+/-1.15 \%$, ND $0.4+/-0.26 \% \mathrm{p}<0.05$ ) (data not shown).

\section{Discussion}

Patients with diabetes mellitus often develop atherosclerosis leading to cardiovascular events. baPWV is a noninvasive and inexpensive method to evaluate vessel wall elasticity and thus can estimate the progress of atherosclerosis without the need for an invasive procedure. Previous reports have claimed positive associations between baPWV and cardiovascular death, cardiovascular event, and stoke in various vascular diseases (13-15). In another report, a positive relation between PWV and intima-media thickness of carotid artery (IMT) was documented (18). Data obtained from baPWV measurement is less diverse when compared to IMT measurement. Since baPWV may be influenced by blood pressure (19), it is critical to match blood pressure to compare baPWV that was validated in this study (see Table 1).

As shown in Fig. 2, candesartan could boost reduction of baPWV compared to $\mathrm{CCB}$ in type 2 diabetes mellitus. On the other hand, there is a report that investigated the comparative effects of candesartan and amlodipine in a monkey atherosclerotic model (20). That report showed that candesartan and amlodipine indicated a similar effect by decreasing the systolic blood pressure approximately $20 \mathrm{mmHg}$, but both medicines did not influence serum cholesterol levels. The ratio of the atherosclerotic area to total area in thoracic aorta was significantly reduced by candesartan, but not by amlodipine. Moreover, another report showed that candesartan but not amlodipine treatment dramatically attenuated the development of atherosclerosis despite a similar reduction in 


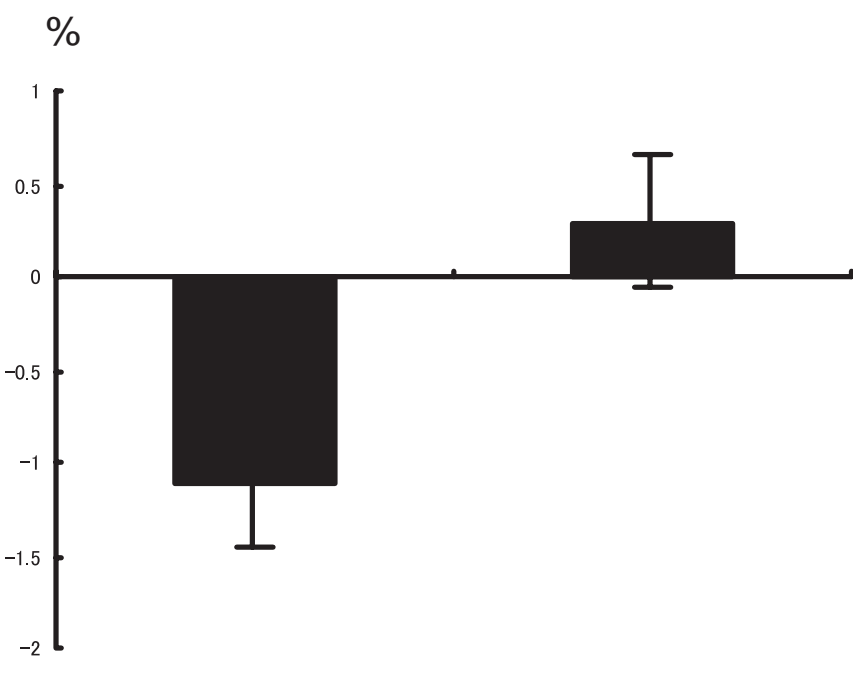

ARB

\section{CCB}

Figure 4. Reduction of HbA1c levels during study period. ARB improved HbA1c levels compared to CCB.

blood pressure in ApoE-deficient mice with a high fat diet (21).

The present data is consistent with these reports. Our PWV data showed that candesartan reduced atherosclerosis without a blood pressure lowering effect. One of reasons for this result may be the recovery of vascular endothelial function. One report showed that candesartan but not amlodipine inhibits the production of reactive oxygen species (21), and another described that ARB stimulated eNOS and caveolin-1 expression and improved coronary microvascular remodeling in normotensive and angiotensin II-induced hypertensive rats (22).

Further, candesartan tended to reduce inflammatory markers (Fig. 3a, b). However, there were no significant differences in inflammatory markers between ARB and CCB. It has been reported that candesartan but not amlodipine inhibited inflammatory gene, MCP-1 or PAI-1, expression in apoE-deficient mice fed a high fat diet (21). Our adhesion assay in vitro supports this report (Fig. 3c). We supposed that the limitation in this study is the low number of participants. If the number of participants would be increased, we may have a significant change between the two groups.

There are some reports which indicate that arterial stiffness is related to inflammation. Some have reported that the reduction of PWV is correlated with the recovery of hsCRP independent of blood pressures $(23,24)$, another showed that CRP is related to measures of arterial wave reflection and stiffness in asymptomatic subjects from the community in a large population study (25). Also, it has been reported hsCRP is associated with PWV in apparently healthy individuals (26). Moreover, there is a report which showed that the expression of adhesion molecules, E-selectin, VCAM-1, and ICAM-1 in blood samples is inhibited when fenofibrate and pioglitazone reduce PWV in obese glucose-tolerant men (27). Although our data had the tendency that inflammation in the blood sample was improved when PWV was reduced, it is insufficient to explain therelation between PWV and inflammation. However, some publications support this relationship.

Next, our data indicated that candesartan decreases HBA1 c (Fig. 4). Angiotensin II inhibits insulin signaling by inhibition of insulin receptor substrate-1 (IRS-1) phosphorylation via AT1 receptor (2) and increased oxidative stress (28). ARB blocks these reactions and improves insulin signaling (29). The VALUE trial showed that ARB inhibits new onset of diabetes mellitus compared to CCB (30). Then, the CASE-J study also showed that candesartan inhibits new onset of diabetes mellitus compared to amlodipine (12), although the CASE-J study included patients without diabetes mellitus. Accordingly, candesartan may recover diabetes mellitus.

Finally, ARB is thought to be the first choice medication for diabetic patients. Our data indicates that candesartan may be suitable for first choice therapy of not only diabetic nephropathy but also macroangiopathy.

\section{Acknowledgement}

We thank Dr. Shunichi Tachibana in Tamananbu Regional Hospital for his assistance.

\section{References}

1. Intensive blood-glucose control with sulphonylureas or insulin compared with conventional treatment and risk of complications in patients with type 2 diabetes (UKPDS 33). UK Prospective Diabetes Study (UKPDS) Group. Lancet 352: 837-853, 1998(Erratum in: Lancet 354:602, 1999).

2. Folli F, Kahn CR, Hansen H, Bouchie JL, Feener EP. Angiotensin II inhibits insulin signaling in aortic smooth muscle cells at multiple levels. A potential role for serine phosphorylation in insulin/ angiotensin II crosstalk. J Clin Invest 100: 2158-2169, 1997.

3. Daugherty A, Manning MW, Cassis LA. Angiotensin II promotes atherosclerotic lesions and aneurysms in apolipoprotein E-deficient mice. J Clin Invest 105: 1605-1612, 2000.

4. Weiss D, Kools JJ, Taylor WR. Angiotensin II-induced hypertension accelerates the development of atherosclerosis in apoE- deficient mice. Circulation 103: 448-454, 2001.

5. Andersson RG, Jacobsson L, Persson K. Angiotensin converting enzyme inhibitors and atherosclerosis. J Physiol Pharmacol 45: 13-25, 1994.

6. Ridker PM, Cushman M, Stampfer MJ, Tracy RP, Hennekens CH. Inflammation, aspirin, and the risk of cardiovascular disease in apparently healthy men. N Engl J Med 336: 973-979, 1997.

7. Ikeda U, Ito T, Shimada K. Interleukin-6 and acute coronary syndrome. Clin Cardiol 24: 701-704, 2001.

8. Ross R. Atherosclerosis--an inflammatory disease. N Engl J Med 340: 115-126, 1999.

9. Bevilacqua MP, Nelson RM, Mannori G, Cecconi O. Endothelialleukocyte adhesion molecules in human disease. Annu Rev Med 45: 361-378, 1994. 
10. Wu L, Iwai M, Nakagami H, et al. Roles of angiotensin II type 2 receptor stimulation associated with selective angiotensin II type 1 receptor blockade with valsartan in the improvement of inflammation-induced vascular injury. Circulation 104: 2716-2721, 2001.

11. Cianchetti S, Del Fiorentino A, Colognato R, Di Stefano R, Franzoni F, Pedrinelli R. Anti-inflammatory and anti-oxidant properties of telmisartan in cultured human umbilical vein endothelial cells. Atherosclerosis 198: 22-28, 2008.

12. Ogihara T, Nakao K, Fukui T, et al. for the Candesartan Antihypertensive Survival Evaluation in Japan Trial Group. Effects of candesartan compared with amlodipine in hypertensive patients with high cardiovascular risks: candesartan antihypertensive survival evaluation in Japan trial. Hypertension 51: 393-398, 2008.

13. Imanishi $R$, Seto $S$, Toda $G$, et al. High brachial-ankle pulse wave velocity is an independent predictor of the presence of coronary artery disease in men. Hypertens Res 27: 71-78, 2004.

14. Ogawa $\mathrm{O}$, Onuma $\mathrm{T}$, Kubo $\mathrm{S}$, Mitsuhashi $\mathrm{N}$, Muramatsu $\mathrm{C}$, Kawamori R. Brachial-ankle pulse wave velocity and symptomatic cerebral infarction in patients with type 2 diabetes: a crosssectional study. Cardiovasc Diabetol 2: 10, 2003.

15. Matsuoka O, Otsuka K, Murakami S, et al. Arterial stiffness independently predicts cardiovascular events in an elderly community - Longitudinal Investigation for the Longevity and Aging in Hokkaido County (LILAC) study. Biomed Pharmacother suppl 1: S40S44, 2005.

16. Kobayashi K, Akishita M, Yu W, Hashimoto M, Ohni M, Toba K. Interrelationship between non-invasive measurements of atherosclerosis: flow-mediated dilation of brachial artery, carotid intimamedia thickness and pulse wave velocity. Atherosclerosis 173: 1318, 2004.

17. Bevilacqua MP, Pober JS, Mendrick DL, Cotran RS, Gimbrone MA Jr. Identification of an inducible endothelial-leukocyte adhesion molecule. Proc Natl Acad Sci U S A 84: 9238-9242, 1987.

18. Taniwaki H, Kawagishi T, Emoto M, et al. Correlation between the intima-media thickness of the carotid artery and aortic pulsewave velocity in patients with type 2 diabetes. Vessel wall properties in type 2 diabetes. Diabetes Care 22: 1851-1857, 1999.

19. Ibata J, Sasaki H, Kakimoto $T$, et al. Cardio-ankle vascular index measures arterial wall stiffness independent of blood pressure.
Diabetes Res Clin Pract 80: 265-270, 2008.

20. Takai S, Jin D, Sakaguchi M, et al. Comparative effects of candesartan and amlodipine in a monkey atherosclerotic model. Hypertens Res 27: 517-522, 2004.

21. Doran DE, Weiss D, Zhang Y, Griendling KK, Taylor WR. Differential effects of AT1 receptor and $\mathrm{Ca} 2+$ channel blockade on atherosclerosis, inflammatory gene expression, and production of reactive oxygen species. Atherosclerosis 195: 39-47, 2007.

22. Kobayashi N, Mori Y, Nakano S, et al. TCV-116 stimulates eNOS and caveolin-1 expression and improves coronary microvascular remodeling in normotensive and angiotensin II-induced hypertensive rats. Atherosclerosis 158: 359-368, 2001.

23. Pietri P, Vyssoulis G, Vlachopoulos C, et al. Relationship between low-grade inflammation and arterial stiffness in patients with essential hypertension. J Hypertens 24: 2231-2238, 2006.

24. Kim JS, Kang TS, Kim JB, et al. Significant association of Creactive protein with arterial stiffness in treated non-diabetic hypertensive patients. Atherosclerosis 192: 401-406, 2007.

25. Kullo IJ, Seward JB, Bailey KR, et al. C-reactive protein is related to arterial wave reflection and stiffness in asymptomatic subjects from the community. Am J Hypertens 18: 1123-1129, 2005.

26. Yasmin MC, Wallace S, Mackenzie IS, Cockcroft JR, Wilkinson IB. C-Reactive Protein is associated with arterial stiffness in apparently healthy individuals. Arterioscler Thromb Vasc Biol 24: 969-974, 2004.

27. Ryan KE, McCance DR, Powell L, McMahon R, Trimble ER. Fenofibrate and pioglitazone improve endothelial function and reduce arterial stiffness in obese glucose tolerant men. Atherosclerosis 194: 123-130, 2007.

28. Ogihara T, Asano T, Ando K, et al. Angiotensin II-induced insulin resistance is associated with enhanced insulin signaling. Hypertension 40: 872-879, 2002.

29. Shiuchi T, Iwai M, Li HS, et al. Angiotensin II type-1 receptor blocker valsartan enhances insulin sensitivity in skeletal muscles of diabetic mice. Hypertension 43: 1003-1010, 2004.

30. Julius S, Kjeldsen SE, Weber M, et al. VALUE trial group. Outcomes in hypertensive patients at high cardiovascular risk treated with regimens based on valsartan or amlodipine: the VALUE randomised trial. Lancet 363: 2022-2031, 2004.

(C) 2008 The Japanese Society of Internal Medicine http://www.naika.or.jp/imindex.html 